\title{
A NOTE ON NONNEGATIVE QUADRATIC ORTHOGONAL BISECTIONAL CURVATURE
}

\author{
YANYAN NIU \\ Abstract. In this note, we give a new proof of the nonnegativity of the \\ scalar curvature under the condition of the nonnegative quadratic orthogonal \\ bisectional curvature.
}

\section{INTRODUCTION}

Let $\left(M^{n}, g\right)$ be a complex $n$-dimensional Kähler manifold with $n \geq 2$. We say that $\left(M^{n}, g\right)$ has nonnegative quadratic orthogonal bisectional curvature (NQOBC, for short) at $p \in M$ if for any unitary frame $\left\{e_{i}\right\}_{i=1}^{n}$ of $T_{p}^{1,0} M$ and real numbers $\left\{\xi^{i}\right\}_{i=1}^{n}$,

$$
\sum_{i \neq j} R_{i \bar{i} \bar{j} \bar{j}}\left(\xi^{i}-\xi^{j}\right)^{2} \geq 0 .
$$

A Kähler manifold $\left(M^{n}, g\right)$ is said to have NQOBC provided that the NQOBC condition (1.1) holds at any point $p \in M$. If the strict inequality in (1.1) holds at any point $p \in M$, provided that not all $\xi^{i}$ are the same, we say $\left(M^{n}, g\right)$ has positive quadratic orthogonal bisectional curvature, denoted as PQOBC.

Recall that $\left(M^{n}, g\right)$ is said to have nonnegative orthogonal bisectional curvature (NOBC for short) if, for any point $q \in M$ and any vectors $X, Y \in T_{q}^{1,0} M$, $R(X, \bar{X}, Y, \bar{Y}) \geq 0$ if $\langle X, Y\rangle=0$. NOBC is a slightly weaker condition than requiring that $M$ has nonnegative bisectional curvature. By the work of Chen [5] and $\mathrm{Gu}$ and Zhang [6, a compact simply connected irreducible Kähler manifold with NOBC is either biholomorphic to $\mathbb{C P}^{n}$ or is isometrically biholomorphic to an irreducible compact Hermitian symmetric space of rank at least 2. Their results extend the generalized Frankel conjecture for compact Kähler manifolds with nonnegative holomorphic bisectional curvature established by Bando [1] and Mok [8].

Obviously, a manifold with NOBC has NQOBC. Particularly, in complex dimension 2, the NQOBC condition is equivalent to that of NOBC. But in general, a Kähler manifold with NQOBC may not have NOBC. In [7], the authors constructed a compact 7-dimensional simply connected homogeneous Kähler manifold with second Betti number $b_{2}=1$ and NQOBC, which does not admit any Kähler metric with NOBC. So the condition NQOBC is weaker than that of NOBC. Recently, Chau and Tam 44 gave the necessary and sufficient conditions for an irreducible Kähler C-space with $b_{2}=1$ to have NQOBC or PQOBC.

Received by the editors December 26, 2012.

2010 Mathematics Subject Classification. Primary 53C20, 53C55.

Key words and phrases. Quadratic orthogonal bisectional curvature.

The author was supported by NSFC (\#11301354).

The author would like to thank Professor L. F. Tam for helpful discussions. 
On a Kähler manifold, for a $(1,1)$ form $\phi$, the Bochner formula asserts

$$
\Delta_{\bar{\partial}} \phi_{\alpha \bar{\beta}}=-\Delta \phi_{\alpha \bar{\beta}}-R_{\alpha \bar{\beta} \gamma \bar{\delta}} \phi_{\delta \bar{\gamma}}+\frac{1}{2}\left(R_{\alpha \bar{\gamma}} \phi_{\gamma \bar{\beta}}+R_{\gamma \bar{\beta}} \phi_{\alpha \bar{\gamma}}\right),
$$

where $\Delta_{\bar{\partial}}$ is the Hodge Laplacian and $\Delta=\frac{1}{2}\left(\nabla_{i} \nabla_{\bar{i}}+\nabla_{\bar{i}} \nabla_{i}\right)$ is the tensor Laplacian. From this, it is easy to obtain that on a compact Kähler manifold with NQOBC, the harmonic $(1,1)$ form must be parallel. One can also reformulate the condition of NQOBC by (1.2), that is, $\left(M^{n}, g\right)$ has NQOBC if for any real $(1,1)$ form $\phi \in \Lambda^{1,1} M$,

$$
R_{\alpha \bar{\beta} \gamma \bar{\delta}} \phi_{\beta \bar{\alpha}} \phi_{\delta \bar{\gamma}}-R_{\alpha \bar{\gamma}} \phi_{\gamma \bar{\beta}} \phi_{\beta \bar{\alpha}} \leq 0 .
$$

A Kähler manifold with NQOBC satisfies some interesting properties. In [9], this condition was first considered. Moreover, the authors proved that on such compact manifolds, any boundary class of the Kähler cone can be represented by a smooth closed $(1,1)$ form which is nonnegative. After that, Chau and Tam [3] proved that under NQOBC the scalar curvature is nonnegative by considering the integration of the bisectional curvature and holomorphic sectional curvature over the unitary group $U(n)$. They also established some rigidity results there. In this note, we give a new proof of their results about scalar curvature. Our proof follows from a lower bound for the sum of two eigenvalues of Ricci curvature on a manifold with NQOBC. The proof here is a bit more direct and only uses elementary algebra. The main result is the following:

Theorem 1.1. Let $\left(M^{n}, g\right)$ be a complete Kähler manifold with $N Q O B C$. Then for any point $p \in M$ and any orthogonal unitary pair $V, W \in T_{p}^{1,0} M$, we have

$$
\operatorname{Ric}(V, \bar{V})+\operatorname{Ric}(W, \bar{W}) \geq 2 R(V, \bar{V}, W, \bar{W}),
$$

which implies that the scalar curvature must be nonnegative and the scalar curvature $S(p)=0$ at point $p$ if and only if for any unitary pair $V, W, R(V, \bar{V}, V, \bar{V})+$ $R(W, \bar{W}, W, \bar{W})=0$ and $R(V, \bar{V}, W, \bar{W})=0$. If $n \geq 3, S(p)=0$ also implies that $R(V, \bar{V}, V, \bar{V})=0$ for all $V \in T_{p}^{1,0}(M)$ and hence $M$ is flat at $p$.

In fact, comparing with Theorem 3.1 in the paper by Chau and Tam [3], the new content is the inequality (1.3).

\section{Proof of Theorem 1.1}

By the definition of NQOBC, we know at any point $p \in M$, for any unitary frame $e_{1}, \cdots, e_{n} \in T_{p}^{1,0} M$ and any real numbers $\left\{\xi_{i}\right\}_{i=1}^{n}$,

$$
\sum_{i \neq j} R_{i \bar{i} \bar{j} \bar{j}}\left(\xi^{i}-\xi^{j}\right)^{2} \geq 0 .
$$

We assume that $k \neq l$. By letting $\xi^{k}=0, \xi^{l}=2, \xi^{j}=1$ for any $j \neq k, l$, then

$$
4 R_{k \bar{k} l \bar{l}}+\sum_{j \neq k, l}\left(R_{k \bar{k} j \bar{j}}+R_{l \bar{l} j \bar{j}}\right) \geq 0 .
$$

By letting $\tilde{e}_{k}=\frac{e_{k}-e_{l}}{\sqrt{2}}, \tilde{e}_{l}=\frac{e_{k}+e_{l}}{\sqrt{2}}, \tilde{e}_{j}=e_{j}$ for $j \neq k, l$, then $\left\{\tilde{e}_{i}\right\}$ is also an orthonormal frame. Hence, by (2.1), we have

$$
4 R\left(\tilde{e}_{k}, \overline{\tilde{e}}_{k}, \tilde{e}_{l}, \overline{\tilde{e}}_{l}\right)+\sum_{j \neq k, l}\left(R\left(\tilde{e}_{k}, \overline{\tilde{e}}_{k}, \tilde{e}_{j}, \overline{\tilde{e}}_{j}\right)+R\left(\tilde{e}_{l}, \overline{\tilde{e}}_{l}, \tilde{e}_{j}, \overline{\tilde{e}}_{j}\right)\right) \geq 0 .
$$


After direct calculations, we get

$$
R_{k \bar{k} k \bar{k}}+R_{l \bar{l} l \bar{l}}-R_{k \bar{l} k \bar{l}}-R_{l \bar{k} l \bar{k}}+\sum_{j \neq k, l}\left(R_{k \bar{k} j \bar{j}}+R_{l \bar{j} j \bar{j}}\right) \geq 0 .
$$

By replacing $e_{l}$ with $\sqrt{-1} e_{l}$, we have

$$
R_{k \bar{k} k \bar{k}}+R_{l \bar{l} \bar{l} \bar{l}}+R_{k \bar{l} k \bar{l}}+R_{l \bar{k} l \bar{k}}+\sum_{j \neq k, l}\left(R_{k \bar{k} j \bar{j}}+R_{l \bar{l} j \bar{j}}\right) \geq 0 .
$$

By summing (2.2) and (2.3), we have

$$
R_{k \bar{k} k \bar{k}}+R_{l \bar{l} l \bar{l}}+\sum_{j \neq k, l}\left(R_{k \bar{k} j \bar{j}}+R_{l \bar{l} j \bar{j}}\right) \geq 0 .
$$

Then,

$$
R_{k \bar{k}}+R_{l \bar{l}}=R_{k \bar{k} k \bar{k}}+R_{l \bar{l} l \bar{l}}+2 R_{k \bar{k} l \bar{l}}+\sum_{j \neq k, l}\left(R_{k \bar{k} j \bar{j}}+R_{l \bar{l} j \bar{j}}\right) \geq 2 R_{k \bar{k} l \bar{l}}
$$

This proves (1.3).

If we take $\xi^{k}=1, \xi^{j}=0$ for any $j \neq k$, then $\sum_{j \neq k} R_{k \bar{k} j \bar{j}} \geq 0$ from (1.1). Then,

$$
S=\frac{1}{n-1} \sum_{1 \leq k<l \leq n}\left(R_{k \bar{k}}+R_{l \bar{l}}\right) \geq \frac{2}{n-1} \sum_{1 \leq k<l \leq n} R_{k \bar{k} l \bar{l}} \geq 0 .
$$

That is, the scalar curvature is nonnegative under NQOBC conditions.

If $S(p)=0$, then $\sum_{1 \leq k<l \leq n} R_{k \bar{k} l \bar{l}}=0$. But $S=\sum_{j} R_{j \bar{j} j \bar{j}}+2 \sum_{1 \leq k<l \leq n} R_{k \bar{k} l \bar{l}}$, so $\sum_{j} R_{j \bar{j} j \bar{j}}=0$. On the other hand, by (2.5), $S=0$ implies that for any $\bar{k} \neq l$,

$$
R_{k \bar{k} k \bar{k}}+R_{l \bar{l} \bar{l}}+\sum_{j \neq k, l}\left(R_{k \bar{k} j \bar{j}}+R_{l \bar{l} j \bar{j}}\right)=0 .
$$

By taking $\xi^{k}=1, \xi^{l}=1, \xi^{j}=0$ for any $j \neq k, l$, from (1.1), we have

$$
\sum_{j \neq k, l}\left(R_{k \bar{k} j \bar{j}}+R_{l \bar{l} j \bar{j}}\right) \geq 0 .
$$

Therefore, for any $k \neq l, R_{k \bar{k} k \bar{k}}+R_{\bar{l} \bar{l} \bar{l}} \leq 0$. So we must have for any $k \neq l$, $R_{k \bar{k} k \bar{k}}+R_{l \bar{l} \bar{l} \bar{l}}=0$ since $\sum_{j} R_{j \bar{j} j \bar{j}}=0$. Then, it is easy to obtain that $R_{k \bar{k} l \bar{l}}=0$ for any $k \neq l$.

Since

$$
S=\frac{1}{n-1} \sum_{k<l}\left(R_{k \bar{k} k \bar{k}}+R_{\bar{l} \bar{l} \bar{l}}\right)+2 \sum_{k<l} R_{k \bar{k} l \bar{l}},
$$

then $S(p)=0$ if $R_{k \bar{k} k \bar{k}}+R_{\bar{l} \bar{l} \bar{l}}=0$ and $R_{k \bar{k} l \bar{l}}=0$.

The last statement follows immediately from 3 .

\section{Some COnSEQuences of (1.3)}

Here we bound the curvature tensor by scalar curvature under the condition NQOBC. Let $(\operatorname{Ric} * g)_{i \bar{j} k \bar{l}}=\frac{1}{2}\left(R_{i \bar{j}} g_{k \bar{l}}+R_{k \bar{l}} g_{i \bar{j}}+R_{i \bar{l}} g_{k \bar{j}}+R_{k \bar{j}} g_{i \bar{l}}\right)$ and $W \doteqdot \operatorname{Ric} *$ $g-\mathrm{Rm}$, where $\mathrm{Rm}$ is the curvature tensor. Note that $W$ satisfies the first and second Bianchi identities. Also, we have the following corollary directly from Theorem 1.1. 
Corollary 3.1. On a Kähler manifold $\left(M^{n}, g\right)$ with $N Q O B C$, for any orthogonal unitary pair of $(1,0)$-vectors $X, Y$, we have

$$
W(X, \bar{X}, Y, \bar{Y}) \geq 0 \text {. }
$$

That is, $W$ has nonnegative orthogonal bisectional curvature.

Also, we have the following estimates bounding the Ricci and the curvature tensor by the scalar curvature.

Corollary 3.2. Suppose $\left(M^{n}, g\right)$ has $N Q O B C$ at $p \in M$. Then,

$$
(n-2) R_{i \bar{j}}+S g_{i \bar{j}} \geq 0
$$

where $S$ is the scalar curvature at $p$. In fact, for any unitary frame $\left\{e_{i}\right\}$,

$$
(n-2) R_{i \bar{i}}+S \geq 2 \sum_{j \neq i} R_{i \bar{i} \bar{j} \bar{j}} .
$$

Moreover, if $n \geq 3$, then

$$
-\frac{1}{n-2} S \leq R_{i \bar{i}} \leq \frac{2 n-3}{n-2} S
$$

and if $i \neq j$, then

$$
\begin{gathered}
-(2 n-3) S \leq R_{i \bar{i} j \bar{j}} \leq \frac{(2 n-3)}{n-2} S, \\
-\frac{(n-1)(2 n-3)+1}{n-2} S \leq R_{\bar{i} \bar{i} \bar{i}} \leq \frac{2 n-3}{n-2} S .
\end{gathered}
$$

In particular, the curvature tensor is bounded in terms of the bound of $S$.

Proof. Let $\left\{e_{i}\right\}$ be a unitary frame. By Theorem 1.1 for any $j \neq i$,

$$
R_{i \bar{i}}+R_{j \bar{j}} \geq 2 R_{i \bar{i} \bar{j} \bar{j}} .
$$

Hence,

$$
(n-1) R_{i \bar{i}}+\sum_{j \neq i} R_{j \bar{j}} \geq 2 \sum_{j \neq i} R_{i \bar{i} j \bar{j}} \geq 0 .
$$

This proves (3.1) and (3.2).

To prove (3.3), the lower bound follows from (3.1). Again by (3.1),

$$
-\frac{n-1}{n-2} S+R_{i \bar{i}} \leq \sum_{j=1}^{n} R_{j \bar{j}}=S .
$$

From this the upper bound in (3.3) follows.

To prove (3.4), by (1.3) and (3.3), we have

$$
2 R_{i \bar{i} k \bar{k}} \leq R_{i \bar{i}}+R_{k \bar{k}} \leq \frac{2(2 n-3)}{n-2} S .
$$

Now

$$
0 \leq \sum_{k \neq i} R_{i \bar{i} k \bar{k}}=R_{i \bar{i} \bar{j} \bar{j}}+\sum_{k \neq i, j} R_{i \bar{i} k \bar{k}} \leq R_{i \bar{i} \bar{j} \bar{j}}+(2 n-3) S .
$$

Hence,

$$
R_{i \bar{i} j \bar{j}} \geq-(2 n-3) S .
$$

Now (3.5) follows from (3.4) and (3.3). 


\section{REFERENCES}

[1] Shigetoshi Bando, On the classification of three-dimensional compact Kaehler manifolds of nonnegative bisectional curvature, J. Differential Geom. 19 (1984), no. 2, 283-297. MR755227 (86i:53042)

[2] R. L. Bishop and S. I. Goldberg, On the second cohomology group of a Kaehler manifold of positive curvature, Proc. Amer. Math. Soc. 16 (1965), 119-122. MR.0172221 (30 \#2441)

[3] Albert Chau and Luen-Fai Tam, On quadratic orthogonal bisectional curvature, J. Differential Geom. 92 (2012), no. 2, 187-200. MR2998670

[4] A. Chau and L. F. Tam, Kähler $C$-spaces and quadratic bisectional curvature, J. Differential Geom. 94 (2013), no. 3, 409-468. MR3080488

[5] X. X. Chen, On Kähler manifolds with positive orthogonal bisectional curvature, Adv. Math. 215 (2007), no. 2, 427-445, DOI 10.1016/j.aim.2006.11.006. MR.2355611(2008h:32029)

[6] HuiLing Gu and ZhuHong Zhang, An extension of Mok's theorem on the generalized Frankel conjecture, Sci. China Math. 53 (2010), no. 5, 1253-1264, DOI 10.1007/s11425-010-0013-y. MR2653275 (2011d:53156)

[7] Q. Li, D. Wu and F. Y. Zheng, An example of compact Kähler manifold with nonnegative quadratic bisectional curvature, Proc. Amer. Math. Soc. 141 (2013), no. 6, 2117-2126. MR.3034437

[8] Ngaiming Mok, The uniformization theorem for compact Kähler manifolds of nonnegative holomorphic bisectional curvature, J. Differential Geom. 27 (1988), no. 2, 179-214. MR.925119 (89d:53115)

[9] Damin Wu, Shing-Tung Yau, and Fangyang Zheng, A degenerate Monge-Ampère equation and the boundary classes of Kähler cones, Math. Res. Lett. 16 (2009), no. 2, 365-374. MR2496750 (2010c:32061)

School of Mathematical Sciences, Capital Normal University, 105 Xisanhuan Beilu, Beijing, 100048, People's Republic of China

E-mail address: yyniukxe@gmail.com 